\title{
Transposition
}

Musique et Sciences Sociales

9| 2021

Musique et sexualité

\section{Paul Edwards, Elodie Grossi \& Paul Schor (dir.), Disorder. Histoire sociale des mouvements punk \& post- punk}

Guichen, éditions Mélanie Seteun, 2019

\section{Sarah Benhaïm}

\section{OpenEdition}

Journals

Édition électronique

URL : http://journals.openedition.org/transposition/5762

DOI : 10.4000/transposition.5762

ISSN : 2110-6134

Éditeur

CRAL - Centre de recherche sur les arts et le langage

Référence électronique

Sarah Benhaïm, «Paul Edwards, Elodie Grossi \& Paul Schor (dir.), Disorder. Histoire sociale des mouvements punk \& post-punk», Transposition [En ligne], 9 | 2021, mis en ligne le 15 décembre 2020 consulté le 23 avril 2021. URL : http://journals.openedition.org/transposition/5762 ; DOI : https:// doi.org/10.4000/transposition.5762

Ce document a été généré automatiquement le 23 avril 2021

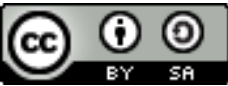

La revue Transposition est mise à disposition selon les termes de la Licence Creative Commons Attribution - Partage dans les Mêmes Conditions 4.0 International. 


\section{Paul Edwards, Elodie Grossi \& Paul Schor (dir.), Disorder. Histoire sociale des mouvements punk \& post-punk}

Guichen, éditions Mélanie Seteun, 2019

Sarah Benhaïm

\section{RÉFÉRENCE}

Paul Edwards, Elodie Grossi \& Paul Schor (dir.), Disorder. Histoire sociale des mouvements punk \& post-punk, Guichen, éditions Mélanie Seteun, 2019, 406 p.

«Si l'on n'écrit pas notre histoire, ce sera, comme d'habitude, les rock-critics et les ennemis qui l'écriront» (p.199). Cette sentence cinglante, énoncée par le manager punk Marsu et reprise en guise de titre pour son entretien figurant dans l'ouvrage, formule à elle seule la complexité de la construction d'un récit académique sur les mouvements punk et post-punk. Il convient en premier lieu de rappeler que ces mouvements ont longtemps constitué un champ peu étudié au sein de la recherche académique, à l'exception notable des cultural studies britanniques ${ }^{1}$ et de quelques recherches plus isolées ${ }^{2}$ menées au moment de leur émergence dans les années 1970 et 1980. Si certains écrits journalistiques ${ }^{3}$ ont été précieux du point de vue de la qualité de l'écriture et de la compilation des sources, ils ont parfois aussi contribué à façonner un récit dominant non sans distorsions et apories, alimentant chez les acteurs du punk un sentiment de dépossession. Force est pourtant de constater qu'un sursaut d'intérêt envers le punk et la nébuleuse post-punk a gagné la recherche ces dernières années. Cette «deuxième vague» internationale, que l'on observe depuis les années 2010, suscite un phénomène saillant qui tient autant à l'investissement progressif des popular music studies par les jeunes chercheurs et chercheuses qu'à l'infiltration progressive de punks dans le champ académique - ces punkademics ${ }^{4}$, pour certains réunis au sein du «Punk Scholars Network $»^{5}$, contribuant ainsi directement à cette punkademia ${ }^{6}$. Si des 
publications notoires paraissent régulièrement depuis ${ }^{7}$, jusqu'au récent Deindustrialisation and Popular Music. Punk and 'Post-Punk' in Manchester, Düsseldorf, Torino and Tampere de Giacomo Bottà ${ }^{8}$, d'autres initiatives importantes témoignent de cette effervescence en contribuant à appréhender de manière plus approfondie et diversifiée ces mouvements musicaux et sociaux, comme la série de colloques internationaux sur le DIY initiée par Paula Guerra au Portugal depuis 2014 ou le projet ANR PIND fondé par Luc Robène et Solveig Serre en 2016.

2 C'est justement à la suite d'un colloque universitaire international organisé les 27 et 28 mars 2015 à Paris, intitulé « Disorder. Histoire sociale des mouvements punk et postpunk $»^{9}$, que l'ouvrage du même titre est publié aux éditions Mélanie Seteun en 2019, assorti d'une couverture délibérément déchirée en clin d'œil à l'esthétique destroy du punk. Édité par Paul Edwards, Élodie Grossi et Paul Schor, cet ouvrage collectif d'environ 400 pages s'inscrit dans une perspective historique et sociale qui vise à proposer une alternative aux récits mythiques du punk et du post-punk façonnés en partie par les médias. Le constat d'une histoire canonisée de ces mouvements se fonde en particulier sur deux points, mis en exergue dans l'introduction. D'abord, une chronologie le plus souvent axée sur une courte période (1975-1978) supposée incarner toute l'ébullition créative et l'authenticité de ce courant musical, qui a pour conséquence d'occulter de nombreuses autres tendances musicales influentes communément rassemblées dans ce pot-pourri que l'on nomme "post-punk». Les connaisseurs et connaisseuses de musiques bruitistes se réjouiront ainsi de cette piqûre de rappel réaffirmant que des groupes comme Cabaret Voltaire et Throbbing Gristle, souvent catégorisés comme "post-punk", ont en réalité commencé à mener leurs expérimentations sonores avant les productions spectaculaires des Sex Pistols. Car le second problème tient justement à la glorification d'une poignée de figures charismatiques et médiatiques: outre l'invisibilisation de pléthore d'autres artistes participant à ces scènes, elle tend à occulter le rôle de tous les acteurs œuvrant collectivement à faire advenir, diffuser et apprécier ces productions musicales.

3 Afin de prendre la distance nécessaire à l'égard d'un point de vue dominant dans le récit historique de ces mouvements, les quatorze contributions scientifiques de l'ouvrage explorent une pluralité de courants musicaux affiliés au punk et au postpunk, disséminés tant géographiquement que chronologiquement, qu'elles analysent au moyen d'une diversité méthodologique et disciplinaire remarquable et représentative du large spectre des sciences sociales. Cette démarche de déconstruction implique également d'interroger le sens et les modalités de transmission, et donc aussi de patrimonialisation, d'un mouvement qui s'est longtemps affirmé comme "no future ». Les directeurs de l'ouvrage n'éludent pas le questionnement épistémologique que pose la manipulation des sources - le plus souvent restées la propriété personnelle et affective de celles et ceux qui en ont été ou en sont les acteurs - et l'archéologie d'un mouvement généralement défiant à l'égard des institutions. Ils proposent ainsi, outre la déclaration d'intention préalable, d'intégrer à l'ouvrage collectif le témoignage de deux acteurs étroitement engagés dans le mouvement en Angleterre et en France: Steve Shepherd, promoteur, conservateur et ancien disquaire réputé dans les milieux punk et post-punk de Liverpool et de Manchester, et Marsu, manager et label de nombreux groupes de la scène punk parisienne des années 1980, dont les iconiques Bérurier Noir.

4 L'entretien du premier, conduit par Edwards, Grossi et Schor, fait justement apparaître une dichotomie dans la définition du punk, entre un punk cristallisé au sein d'une 
période circonscrite et un « esprit du punk » qui transcende les catégories stylistiques, les chronologies usitées et les récits standardisés - cette "dynamique des idées " contre-culturelles, la diversité des pratiques artistiques originelles et l'autonomisation économique de cet underground musical ayant imprégné parallèlement et successivement les courants post-punk et permis un échappatoire à l'uniformité et à la redondance d'un punk en proie à une "image-cliché». Si Shepherd est loin de contredire l'importance des groupes canoniques du punk britannique, il livre une critique acerbe au sujet de la mythification opérée par certains auteurs ${ }^{10}$, appelant ainsi à déconstruire certaines contre-vérités. L'entretien que Schor a réalisé avec Marsu fait montre de la même sévérité, celui-ci désignant comme "ennemis » les auteurs ayant sorti des ouvrages entre "la mythification, l'autoglorification et le révisionnisme " (p. 233), déplorant parallèlement le retard pris par la France en matière de recherche, qui nécessiterait selon lui de déconstruire un récit conçu comme nostalgique, passéiste et non fidèle aux postures et discours initiaux. Lui aussi souligne la porosité de ces scènes en France en rendant compte, d'une part, du glissement du punk vers une scène « alterno » plus festive et son impact sur la fidélité d'un public originellement uni sous l'égide d'une approche plus révolutionnaire, et d'autre part, de la porosité des circuits punk avec ceux du hip hop, de la techno et de l'électro, recentrant la problématique sur les connexions entre les scènes de niche et l'accès à l'industrie. Au-delà de cette volonté de réhabilitation d'une vérité historique et des rancœurs à l'égard de ce qui est vécu comme une dépossession de leur histoire, les deux entretiens livrent des témoignages fort intéressants sur la culture de la masculinité, sur l'importance du paraître, sur la place des minorités racisées et sexualisées, sur les classes sociales et la marginalité, entre culture ouvrière et bohème issue des écoles d'art («ce sont des déclassés, des décalés ou des déphasés ", p. 215).

5 Les chapitres scientifiques annoncés en introduction comme étant répartis selon quatre parties thématiques ne se trouvent, étrangement, pas organisés dans l'ouvrage selon cet ordre effectif. La première partie met au premier plan l'importance des réseaux dans la constitution de ces mouvements, conçus comme circulation d'agents humains et non-humains. Soucieux de comprendre les origines du monde punk anglais à partir des acteurs précurseurs présents dans plusieurs foyers de Londres et de Manchester entre 1975 et 1976, Nick Crossley schématise les collaborations musicales identifiées afin d'évaluer la densité et la compacité des réseaux. Pour ce faire, l'auteur s'intéresse tant aux processus de recrutement des réseaux qu'aux événements catalyseurs, tels les concerts des Sex Pistols. La production abondante de fanzines, en tant que marqueurs typiques d'une presse underground et amateure, a également joué un rôle prépondérant dans la construction d'un réseau mais aussi de toute évidence, d'un éthos punk. Après avoir brièvement retracé l'histoire de ce médium et mis en exergue le rôle du vocabulaire et de l'imagerie dans la caractérisation du punk vis-à-vis de la société et de l'industrie musicale, Matthew Worley se concentre sur les expressions politiques véhiculées à travers ce support entre 1976 et 1984. Agissant comme lien privilégié entre groupes, labels, salles et fans, les fanzines sont vecteurs d'une critique du star-system et d'un refus de l'exploitation médiatique, en plus de s'intéresser à l'autonomie, au genre et aux inégalités, oscillant autour d'idées empruntes aux gauches et à l'expérimentation anarchiste. Samuel Étienne se penche pour sa part sur les stratégies éditoriales des fanzines punk. On apprend ainsi que l'autonomisation de cette presse underground n'a rien d'une évidence. À la difficulté d'une distribution au-delà du cercle local, que les premiers fanzines punks avaient d'abord éprouvée en distribuant 
via les NMPP faute de réseau alternatif déjà existant, répond ensuite l'ingéniosité des acteurs à créer des "micro-alliances stratégiques": endossant le rôle de "réseaux sociaux analogiques", les fanzines révèlent en effet un réseau informel progressivement tissé entre les distro alternatives mobiles, les disquaires indépendants et les ventes directes, mettant au premier plan les interactions sociales. On le constate aussi à la lecture du chapitre de Christophe Broqua et Vincent Douris, au sujet cette fois des musiques "post-industrielles »: fanzines, labels, distro et disquaires forment un réseau poreux avec celui du punk à travers le partage de pratiques DIY, malgré une esthétique sombre et une mise en scène de l'ambiguïté dans le discours politique. Les auteurs mettent l'accent sur les pratiques d'échanges épistolaires de la scène indus "pré-virtuelle »- une spécificité qui concerne également les musiques noise ${ }^{11}$ - sans lesquelles ces productions issues de micro-scènes décentralisées n'auraient pu être connues du public ni entretenir le fantasme collectif d'une vaste scène internationale.

6 La seconde partie explore, quant à elle, la question des transferts culturels et des circulations transnationales du punk et du post-punk. Pierre Raboud s'intéresse notamment à l'exemple de l'appropriation du punk en Suisse entre 1976 et 1982. Le punk suisse s'accompagne dans un premier temps d'un faible degré de politisation marqué par l'absence dans les paroles des problématiques sociétales typiques du punk britannique, en faveur de l'expression d'un ennui de la jeunesse urbaine que l'auteur associe au conservatisme moral et culturel helvétique. Si la Suisse a été peu touchée par le chômage de masse à la suite de la crise économique des années 1970, elle est traversée par un certain nombre de problèmes politiques et culturels qui incluent criminalisation des opposants et politiques culturelles élitistes peu inclusives de la jeunesse, notamment en matière de musiques actuelles, ayant pour incidence un ralliement progressif des punks à un plus vaste mouvement social et revendicatif de la jeunesse des années 1980. Au cœur cette fois-ci du Milan de ces mêmes années 1980, Simone Tosoni livre les résultats d'une enquête menée sur la scène gothique (dark en italien). À partir du concept d'enactment, celui-ci s'intéresse à la manière dont s'effectuent les différentes appropriations d'un canon subculturel anglais véhiculé par les médias, dont il identifie trois adaptations locales: une version marquée par l'activisme politique autour des thèmes de l'autogestion, de la religion, du genre et des sexualités, avec l'exemple du collectif Creature Simili ; une autre version politiquement désabusée portée sur l'affirmation de soi et sur le rejet des valeurs dominantes, associée à la culture club italienne et suisse; et une troisième version typique de l'arrière-pays milanais, dont le sentiment d'appartenance symbolique à une grande communauté dark est caractérisée par la participation active aux fanzines ou aux concerts. Djordje Sredanovic s'intéresse également au cas italien à partir du groupe de punk/new wave CCCP, ancré dans une tradition culturelle rurale et communiste du Nord, qu'il compare aux groupes punks yougoslaves aux influences plus occidentales et urbaines. L'auteur s'interroge sur les inspirations imaginaires mutuelles, tantôt musicales, tantôt culturelles, entre ces pays de l'Ouest et de l'Est de l'Europe, en questionnant les concepts de désethnicisation et de réethnicisation. Enfin, les tensions que peuvent susciter les régimes autoritaires sur une liberté d'expression propre au punk sont spécialement manifestes dans le cas de la Chine, nous le rappelle le chapitre de Nathanel Amar. Ce sont ici les relations entre la musique «non-officielle » et la police qui sont explorées : après un retour historique bienvenu sur les censures artistiques du rock depuis Tian'anmen, l'auteur prête attention aux stratégies de contournement 
mises en place par les punks pour s'opposer et négocier une répression intensifiée depuis l'accession au pouvoir de Xi Jinping.

7 L'importance des espaces dans la définition des identités musicales ne pourrait être négligée, comme l'a montré de manière emblématique le travail de Sara Cohen. Il s'agit précisément de la direction empruntée dans la troisième partie de cet ouvrage, que Michael Stewart Foley introduit avec la ville de San Francisco, historiquement marquée par les revendications contre-culturelles. La scène punk locale est longtemps restée sous-estimée dans le champ académique, alors même qu'elle détonne par son engagement politique particulièrement prononcé. Au-delà de certains groupes emblématiques comme Dead Kennedys, Flipper ou Tuxedomoon, célèbres pour avoir dénoncé les répressions policières et le militarisme étasunien, le punk a constitué à San Francisco un espace d'engagement en faveur de la lutte contre les inégalités et l'exclusion des populations marginalisées, en défendant une culture participative empreinte à la fois d'idées issues de la nouvelle gauche et d'un éthos utopique des années 1960. La topologie d'un lieu peut par ailleurs être porteuse de sens: Daniel Makagon expose ainsi les résultats de son ethnographie menée dans les sous-sols de maisons individuelles de Chicago, investies par de nombreux concerts informels dont le réseau contribue à la création d'une scène DIY. Habituellement délaissée, la cave constitue ici un espace aux frontières brouillées, entre espace semi-public et résidence privée. Si la distinction par l'auteur de deux tendances dans l'investissement de ces maisons punk nous apparait pour le moins binaire - en ne caricaturant que légèrement, d'un côté le punk propre, bien organisé et responsable, d'un autre le punk "destructeur ", rebelle et crado -, l'investissement collectif de ces résidents s'avère au demeurant décisif dans la création d'une dynamique culturelle locale. L'image d'une ville peut également hériter de succès musicaux fondateurs et mythiques, chers au tourisme municipal. C'est le cas de Glasgow, attitrée "ville créative de musique " par l'UNESCO et « capitale musicale $~_{12}$ de l'Écosse. Si certains groupes internationalement reconnus comme Franz Ferdinand ou Mogwai ont plus récemment jeté la lumière sur la ville, Laurence Estanove revient sur le mythe associé au label Postcard, qui a réussi dès le tournant des années 1980 à détourner la focale médiatique de Londres avec des sorties influentes mêlant pop et éthos punk, à l'image d'Orange Juice, Josef $\mathrm{K}$ ou Aztec Camera. Enfin, un espace peut, d'une toute autre manière, jouir d'un capital symbolique particulier auprès des acteurs du punk lorsqu'il est le lieu d'une "économie de l'underground social ». Le wagon, squat actif à Saint-Brieuc de 1997 à 2004, au sein duquel Djemila Zeneidi a mené une enquête ethnographique passionnante, est un hautlieu du punk qui concentre une forme d'expérience totale de l'être et de l'agir punk. Ce lieu-refuge implique pour ses habitant•es de « rompre avec la norme pour redéfinir sa vie en fonction de ses propres priorités et recouvrer une forme de liberté entravée par des conditions de vie précaires" (p. 389). Le punk est ici le territoire d'une reconnaissance tout autant que le véhicule d'une critique sociale, dont le sentiment de révolte profond s'incarne par les leviers de l'action dans des luttes politiques et identitaires contre toutes les formes d'exclusion et de catégories exogènes dont sont affublés les individus marginalisés.

8 Ce sont finalement les trajectoires des acteurs qui se trouvent explorées par les deux chapitres restants. Pierig Humeau livre ainsi les résultats d'une enquête sociologique (notamment quantitative) menée auprès de participants dans le Grand Ouest français, avec pour objectif d'identifier le parcours et les conditions sociales d'entrée dans le monde punk de ces jeunes, le plus souvent issus de classes populaires. Proches en de 
nombreux points du profil observé par Zeineidi, les tensions intrafamiliales et les rapports conflictuels au monde du travail les ont conduits à partager une socialité et une pratique musicale offrant la possibilité de construire un «nous » collectif, où la culture punk apparaît finalement comme une «requalification symbolique de la précarité » (p. 250) où prime la politisation par la pratique. Le chapitre de Thomas Loué propose quant à lui une analyse monographique du groupe français OTH (1976-1991) réalisée à partir d'un réservoir documentaire historique et ethnologique. L'auteur questionne ici l'identité du groupe au regard de celle de ses membres et explore l'organisation et la territorialisation des tournées, en s'intéressant tant aux socialités qu'aux expressions esthétiques.

9 En somme, la diversité temporelle et spatiale de ces études de cas ainsi que la pluralité des méthodologies exposées composent ici un ouvrage collectif passionnant qui rend compte de la complexité de ces mouvements. On pourrait toutefois regretter que la question des catégories stylistiques soit trop rapidement évacuée (afin de «s'affranchir » de leurs barrières et de ne pas «s'enfermer dans les étiquettes » p. 14), alors même que celles-ci sont très souvent employées et négociées dans les discours et dans les pratiques des acteurs, y compris lorsqu'elles sont justement rejetées ; ainsi, un chapitre analysant ces constructions catégorielles aurait été utile et sans doute plus convaincant pour soutenir l'argumentaire préalable dénonçant leur étroitesse. Reléguer les questions stylistiques aux seules compétences de la musicologie, comme suggéré en introduction, pourrait par ailleurs laisser supposer en filigrane - et de manière paradoxale au regard de la déclaration d'intention scientifique de l'ouvrage que la musicologie ne serait pas une science sociale, ou bien que les débats catégoriels relèveraient nécessairement de l'esthétique. Mais en dehors de ce point de discussion, Disorder apporte assurément une contribution française de grande qualité au champ des popular music studies en analysant avec une attention fine des phénomènes sociaux qui définissent, témoignent et construisent les cultures punk et post-punk d'hier et d'aujourd'hui.

\section{NOTES}

1. HEBDIGE Dick, Sous-culture. Le sens du style, Paris, Zones, 2008 ; LAING Dave, One Chord Wonders. Power and Meaning in Punk Rock, PM Press, 2015.

2. Pour l'exemple de la France, cf. MIGNON Patrick, « Figures urbaines du quotidien : l'effet punk », Esprit, 1978, p. 31-33; DELAPORTE Yves, «Teddies, rockers punk \& cie: quelques codes vestimentaires urbains ", L'Homme, vol. 22, no 4, 1982, p. 49-62 ; ROUÉ Marie, « La punkitude, ou un certain dandysme ", Anthropologie et Sociétés, vol. 10, no 2, 1986, p. 37-55.

3. Cf. par exemple les ouvrages emblématiques de REYNOLDS Simon, Rip it up and start again. Postpunk 1978-1984, 2014 ; MARCUS Greil, Lipstick Traces. Une histoire secrète du vingtième siècle, Paris, Gallimard, 1998 ; SAVAGE Jon, The England's Dreaming Tapes, Paris, Allia, 2011.

4. FURNESS Zach, Punkademics: the Basement Show in the Ivory Tower, Wivenhoe [England]; Brooklyn [New York, N.Y.]; Brooklyn, NY, Minor Compositions, 2012.

5. https://www.punkscholarsnetwork.com 
6. BUSZEK Maria Elena, « Punkademia », American Quarterly, vol. 65, no 2, 2013, p. 413-423.

7. Pour les références anglo-saxonnes emblématiques, citons BESTLEY Russell et oGG Alex, The art of punk, Omnibus Press, 2014 ; GORDON Alastair, Crass Reflections, London, AK Press, 2016 ; DALE Pete, Anyone Can Do it. Empowerment, Tradition and the Punk Underground, Routledge, 2016 ; DINES Mike et WORLEY Matthew, The aesthetic of our anger: anarcho-punk, politics and music, Minor Compositions, 2016 ; BESTLEY Russ, DINES Mike, GORDON Alastair et GUERRA Paula, The Punk Reader Research. Transmissions From the Local and the Global, Intellect Ltd, 2019. Pour un exemple français, citons un numéro de la revue Volume! dirigé par ROBÈNE Luc et SERRE Solveig, La scène punk en France (1976-2016), Guichen (Ille-et-Vilaine), Editions Seteun, 2017.

8. воттÀ Giacomo, Deindustrialisation and Popular Music. Punk and "Post-Punk» in Manchester, Düsseldorf, Torino and Tampere, Lanham, Rowman \& Littlefield Publishing Group, 2020.

9. Ce colloque a été organisé par Paul Edwards, Sébastien Greppo, Élodie Grossi, Travis A. Jackson et Paul Schor avec le soutien du CHCSC, LARCA, Sorbonne Paris Cité et le CNRS. De plus amples informations sur l'événement peuvent être consultées sur le site internet créé à cette occasion : http://colloquepunkpostpunk2015.blogspot.com

10. Steve Shepherd fait par exemple explicitement référence au journaliste et manager des Ramones, Danny Fields.

11. BENHAÏM Sarah, « Noise records as noise culture: DIY practices, aesthetics and trades », BENNETT Andy, GUERRA Paula (dir.), DIY Cultures and Underground Music Scenes, London, Routledge, 2018, p. 112-124.

12. https://fr.unesco.org/creative-cities/glasgow Consulté le 16 juillet 2020.

\section{AUTEURS}

\section{SARAH BENHAÏM}

Sarah Benhaïm est docteure en musique et sciences sociales (CRAL/EHESS). Ses recherches portent sur la musique noise, les pratiques artistiques expérimentales et le Do It Yourself (DIY). Actuellement ATER en musicologie (musiques actuelles/sociologie de la musique) à l'Université de Tours, elle a également enseigné la théorie de l'art et l'histoire du graphisme en écoles d'art. Elle est membre du comité de rédaction de la revue Transposition. Musique et sciences sociales et membre du collège d'experts de la commission Musique de la DRAC Île-de-France. 